\title{
Fields Connected with Particles in Terms of Complex-Riemannian Geometry*
}

\author{
Julian Ławrynowicz \\ Institute of Mathematics, Polish Academy of Sciences, The Lódź Branch
}

Katarzyna Kędzia and Leszek Wojtczak

Institute of Physics, University of Łódź

Z. Naturforsch. 45a, 81-94 (1990); received November 2, 1988

\begin{abstract}
A complex analytical method of solving the generalised Dirac-Maxwell system has recently been proposed by two of us for a certain class of complex Riemannian metrics. The Dirac equation without the field potential in such a metric appeared to be equivalent to the Dirac-Maxwell system including the field potentials produced by the currents of a particle in question. The method proposed is connected with applying the Fourier transform with respect to the electric charge treated as a variable, with the consideration of the mass as an eigenvalue, and with solving suitable convolution equations. In the present research an explicit calculation based on linearization of the spinor connections is given. The conditions for the motion are interpreted as a starting point to seek selection rules for curved space-times corresponding to actually existing particles. Then the same method is applied to solids. Namely, by a suitable transformation of the configuration space in terms of elements of the interaction matrix corresponding to the Coulomb, exchange, and dipole integrals, the interaction term in the hamiltonian becomes zero, thus leading to experimentally verificable formulae for the autocorrelation time.
\end{abstract}

\section{Introduction}

Following the previous papers by two of us $[1,2]$ concerning the description of elementary particles in terms of real- and complex-Riemannian manifolds, here we deal with the construction of fields connected with particles and regarded as a deformation of the particle space, thus providing a natural continuation of [3]. By analogy to the general relativity, where the gravitational field and the space curvature are tightly related, the appearance of a particle determines the space geometry whose properties reflect the particle properties and describe the fields produced by them.

In the quantum field theory the particles and fields are described by the Dirac and Maxwell equations, forming a self-consistent system with respect to the potentials and currents. In this case the Dirac equation without the field potential, but considered in terms of complex-Riemannian geometry, is equivalent to the Dirac-Maxwell system including the field potentials produced by the currents of a particle in question. Taking into account the analogy to the gravitational field mentioned above, the connection describing the particle geometry can be interpreted as

* Research supported by CPBP 01.08 .

Reprint requests to Prof. Julian Ławrynowicz, ul. Krzywickiego 31, PL-90-149 Łódź, Poland. a field produced by a considered particle. Thus, the connection as well as the geometry can be found by means of the Maxwell equations whose solutions are electromagnetic potentials produced by the currents taken in a considered geometry.

The solutions of the Dirac-Maxwell system have been found by generalising for certain curved spacetimes $[4,1]$ the method suggested by Gaveau and Laville [5]. This method is also extended in the case of the present considerations (following [1]) by treating the mass in the uniform way as the charge. More precisely, in [3] the electric charge $\underline{e}$ has been treated as a real variable whose conjugate variable $\varepsilon$ is considered the real part of the complex variable $\varepsilon+i \theta$. This means that the space-time in question has been treated as a typical fibre in the fibre bundle generated by the spectrum of all possible charges. In the present contribution to this question we use uniformly the charge and the mass as independent variables investigated in the direction of determining the spectrum of all possible electric charges and all possible masses. As far as the mass is concerned, it is treated as an eigenvalue $\underline{m}$ of the wave equation resulting from the DiracMaxwell system.

In general, the connection constructed of the basis of the space-time metric is chosen in the complex form since the interactions described by this connection can 
be related at least to the electromagnetic as well as nuclear forces. This means that the elements of the metric are also of the complex form and their real and imaginary parts correspond to different types of interaction fields. In the light of this remark the DiracMaxwell system can be regarded as a generalized system of coupled equations for particles and fields including the Maxwell and Yang-Mills fields.

An explicit calculation based upon linearization of the spinor connections (the generalised Christoffel symbols) is given. Consequently, the generalised scheme of the solution of the Dirac-Maxwell system in a complex-Riemannian geometry is taken as a tool for seeking selection rules for curved space-times corresponding to particles existing in reality.

Finally, it is observed that the method has its macroscopic counterpart for crystals: thin films and bulks as well. A suitable transformation of the interaction matrix corresponding to the Coulomb, exchange, and dipole integrals can, on one hand, let the interaction term in the hamiltonian be zero and, on the other hand, can be interpreted as a transformation of the configuration space, as announced in [6], Sect. 3, yielding - in terms of changing the topology and the Riemannian structure of the space - a fictitious change of the (in)homogeneity of the sample in question. This leads to relatively simple formulae for the correlation function and so to experimentally verificable formulae for the autocorrelation time. In particular, the soliton case for monoatomic layers close to the surface is clearly distinguished.

\section{The Dirac Field and the Electromagnetic Field}

In the Minkowski space-time $\mathbb{M}_{0}$, treated as a space of observations for a given particle, the Dirac equation in the presence of a self-electromagnetic field $\boldsymbol{A}$ reads

$$
D_{0} \psi_{0}=0, \quad \square A=j_{0}, \quad \operatorname{Div} \boldsymbol{A}=0,
$$

where, locally, $D_{0}=\gamma_{0}^{k}\left[\left(\partial / \partial x^{k}\right)+i \underline{e} A_{k}\right]+\underline{m} I_{4}$. Here $i$ is the imaginary unit, while $\underline{m}=\underline{m}_{0} \mathrm{c} / \hbar$ and $\underline{e}=\underline{e}_{0} / \hbar c$, where $\underline{m}_{0}$ and $\underline{e}_{0}$ denote the rest mass and electric charge of the particle in question, respectively, with the usual meaning of $c$ and $\hbar$. The Einstein summation convention is applied throughout the paper. We treat $\psi_{0}: \mathbb{M}_{0} \rightarrow \mathbb{C}^{4}$ as a spinor in the usual sense [7], while $\gamma_{0}^{k}$ denote the Dirac matrices, obtained by the commutation rules $\gamma_{0}^{j} \gamma_{0}^{k}+\gamma_{0}^{k} \gamma_{0}^{j}=2 \eta^{j k} I_{4}$, where $\eta$ denotes the Minkowski metric and $I_{4}$ the identity $4 \times 4$-matrix.
In particular, we may take the representation

$$
\gamma_{0}^{j}=-i\left[\begin{array}{cc}
0_{2} & \sigma_{j} \\
\sigma_{j} & 0_{2}
\end{array}\right], \quad j=1,2,3 ; \quad \gamma_{0}^{0}=-i\left[\begin{array}{rr}
I_{2} & 0_{2} \\
O_{2} & -I_{2}
\end{array}\right],
$$

where $\sigma_{j}$ are the Pauli matrices and $0_{m}$ is the zero $m \times m$-matrix. The symbol $\boldsymbol{j}_{0}$ in (1) denotes the current generated by $\psi_{0}$ :

$$
j_{0}=\underline{e} \bar{\psi}_{0}^{j} \gamma_{0 j k} \psi_{0}^{k}, \quad \gamma_{0 j k}=\left(\gamma_{0 j k}^{1}, \gamma_{0 j k}^{2}, \gamma_{0 j k}^{2}, \gamma_{0 j k}^{0}\right),
$$

where $\bar{\psi}_{0}^{j}$ stands for the complex conjugate of $\psi_{0}^{j}$.

Still, following Kałuża and Klein [8], gravity and electromagnetism can be unified by making use of five dimensions which are the four-dimensional spacetime $\mathbb{M}_{0}$ and the one-dimensional space which, compactified, as $S_{1}$, gives rise to a $U$ (1) gauge symmetry [9]. Following [10], Sect. 6, consider the Clifford matrices $\tilde{\gamma}_{\mu}, \mu=1,2,3,0$, corresponding to the purely imaginary Majorana representation of the Clifford algebra $C^{(3,1)}$. Then $\tilde{\gamma}_{\mu}$ can be expressed by the usual 4-dimensional Majorana representation $\gamma_{\mu}, \mu=$ 1, 2, 3, 0:

$$
\begin{array}{ll}
\tilde{\gamma}_{\mu}=\left[\begin{array}{rr}
0_{4} & \gamma_{\mu} \\
-\gamma_{4} & 0_{4}
\end{array}\right] ; & \gamma_{0}=i\left[\begin{array}{rr}
0_{2} & \theta_{0} \\
-\theta_{0} & 0_{2}
\end{array}\right], \\
\gamma_{1}=i\left[\begin{array}{ll}
0_{2} & \theta_{1} \\
\theta_{1} & 0_{2}
\end{array}\right], & \gamma_{2}=i\left[\begin{array}{rr}
-\theta_{0} & 0_{2} \\
0_{2} & \theta_{0}
\end{array}\right], \quad \gamma_{3}=i\left[\begin{array}{ll}
0_{2} & \theta_{3} \\
\theta_{3} & 0_{2}
\end{array}\right] ; \\
\theta_{0}=\left[\begin{array}{ll}
1 & 0 \\
0 & 1
\end{array}\right], & \theta_{1}=\left[\begin{array}{rr}
0 & -1 \\
-1 & 0
\end{array}\right], \quad \theta_{3}=\left[\begin{array}{rr}
-1 & 0 \\
0 & 1
\end{array}\right] .
\end{array}
$$

The matrices $\tilde{\gamma}_{\mu}$ can be "diagonalized" with help of the following transformation $\mathbf{S}$ :

$$
\mathbf{S} \gamma_{\mu} \mathbf{S}^{-1}=-i\left[\begin{array}{rr}
\gamma_{\mu} & 0_{4} \\
O_{4} & -\gamma_{\mu}
\end{array}\right], \quad \mathbf{S}=2^{-1 / 2}\left[\begin{array}{cc}
I_{4} & i I_{4} \\
i I_{4} & I_{4}
\end{array}\right] \text {. }
$$

As remarked in [10], $\mathbf{S}$ fully determines the transformations shown by arrows in the list of four possible mathematical descriptions of the field in question:

$$
\begin{aligned}
& \left.\left(C^{(3,1)},\left[\begin{array}{rr}
I_{4} & 0_{4} \\
O_{4} & -I_{4}
\end{array}\right]\right) \leftrightarrow C^{(0,4)},\left[\begin{array}{rr}
O_{4} & I_{4} \\
-I_{4} & 0_{4}
\end{array}\right]\right) \\
& \text { (SO-hyperbolic case) } \\
& \left(C^{(3,1)},\left[\begin{array}{rr}
O_{4} & I_{4} \\
-I_{4} & 0_{4}
\end{array}\right]\right) \leftrightarrow\left(C^{(0,4)},\left[\begin{array}{rr}
I_{4} & 0_{4} \\
O_{4} & -I_{4}
\end{array}\right]\right) .
\end{aligned}
$$

$$
\text { (symplectic case) }
$$

$$
\text { (SO-elliptic case) }
$$

The notions of SO-ellipticity and SO-hyperbolicity refer to the groups $\mathrm{SO}(0, s)$ and $\mathrm{SO}(1, s)$, and are defined in [10]. 
Confining ourselves to the symplectic cases in the scheme (3), we decompose $\psi_{0}$ in (1) into two four-dimensional spinors $\psi_{+}$and $\psi_{-}$so that

$$
\boldsymbol{\psi}_{0}=2^{-1 / 2}\left(\psi_{+}+i \psi_{-}\right), \quad \mathbf{S}\left[\begin{array}{l}
\psi_{+} \\
\psi_{-}
\end{array}\right]=\left[\begin{array}{l}
\psi_{0} \\
\bar{\psi}_{0}
\end{array}\right]
$$

After the compactification the original space-time has to be replaced by a fibre bundle $\mathbf{B}_{\mathbf{M}}, \mathbb{M}=\mathbb{M}_{0} \times S_{1}$, with a four-dimensional base space of index 1 or 3 say: $\mathbb{M}_{0}$, and with a one-dimensional compact typical fibre - say: $S_{1}$. The vacuum solutions corresponding to $\mathbf{B}_{\mathbb{M}}$ are obtained from the equations of the gravitational field with the energy-momentum tensor zero. Thus, in the vacuum case the pseudoriemannian tensor of $\mathbf{B}_{M}$ has the form

$\left[g_{a b}^{0}\right]=\left[\begin{array}{cc}{\left[\eta_{\mu v}\right]} & 0 \\ 0^{T} & 1\end{array}\right], \quad 0$ being the zero $4 \times 1$-matrix,

where, as before, $\eta$ denotes the Minkowski metric (we may take the anti-Minkowski metric as well). Then the Kałuża-Klein ansatz (set-up) says that for the low energy theory (zero modes) the pseudo-riemannian tensor of $\mathbf{B}_{\mathbf{M}}$ has the form

$$
\left[g_{a b}\right]=\left[\begin{array}{cc}
{\left[\eta_{\mu \nu}+\underline{e}^{2} A_{\mu} A_{v}\right]} & {\left[-\underline{e} A_{\mu}\right]} \\
{\left[-\underline{e} A_{v}\right]} & 1
\end{array}\right] .
$$

If we denote by $R$ the radius of $S^{1}$, then the above metric is invariant under any transformation of the form

$\boldsymbol{x}^{\prime}=\boldsymbol{x}, \quad \tau^{\prime}=\tau+R \phi(x), \quad A_{\mu}^{\prime}=A_{\mu}-(R / \underline{e}) \partial_{\mu} \phi(x)$,

where $\partial_{\mu}=\partial / \partial x^{\mu}$. The Dirac equation in (1) becomes

$$
\left\{-\gamma_{\mu}\left[\partial^{\mu}-i(\underline{e} / R) A^{\mu}\right]+\partial^{\tau} I_{4}\right\} \psi_{0}(\boldsymbol{x}, \tau)=0 .
$$

By the transversality condition $\partial^{\tau} \psi_{0}=(i / R) \psi_{0}$ we have $\psi_{0}(\boldsymbol{x}, \tau)=\psi_{0}(\boldsymbol{x}) \exp [(i / R) \tau]$ so that

$$
\left\{-\gamma_{\mu}\left[\partial^{\mu}-i(\underline{e} / R) A^{\mu}\right]+(i / R) I_{4}\right\} \psi_{0}(x)=0 .
$$

Consequently, in the symplectic cases in the scheme (3) the Dirac equation (6) describes the Dirac field of mass $\sim 1 / R$, interacting with the electromagnetic field. The SO-hyperbolic and SO-elliptic cases in the scheme (3) correspond to the space of observations and the space of the particle, respectively. The transformation $\mathbf{S}$ in (2) fully determines the transformation between these spaces, defined non-effectively in [2] by formulae (2.2).

The five-dimensional Kałuża-Klein theory has been analysed here as a toy model of more realistic theories in which the scheme (3) is to be replaced by

$$
\begin{aligned}
& \left(C^{(8 k+3,1)},\left[\begin{array}{rr}
I_{\frac{1}{2} n} & O_{\frac{1}{2} n} \\
O_{\frac{1}{2} n} & -I_{\frac{1}{2} n}
\end{array}\right]\right) \leftrightarrow\left(C^{(0,8 k+4)},\left[\begin{array}{rr}
O_{\frac{1}{2} n} I_{\frac{1}{2} n} \\
-I_{\frac{1}{2} n} & O_{\frac{1}{2} n}
\end{array}\right]\right) \\
& \text { (SO-hyperbolic case) } \\
& \left(C^{(8 k+3,1)},\left[\begin{array}{rr}
O_{\frac{1}{2} n} & I_{\frac{1}{2} n} \\
-I_{\frac{1}{2} n} & O_{\frac{1}{2} n}
\end{array}\right]\right) \leftrightarrow\left(C^{(0,8 k+4)},\left[\begin{array}{rr}
I_{\frac{1}{2} n} & O_{\frac{1}{2} n} \\
O_{\frac{1}{2} n} & -I_{\frac{1}{2} n}
\end{array}\right]\right)
\end{aligned}
$$

(symplectic case)

(SO-elliptic case)

with $n=2^{4 k+3}, k=0,1,2, \ldots$, or by

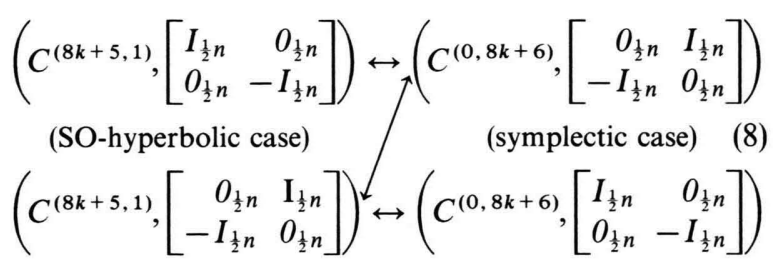

(symplectic case)

(SO-elliptic case)

with $n=2^{4 k+4}, k=0,1,2, \ldots$.

\section{A Complex-analytical Method and its Physical Interpretation}

The mathematical description of the SO-elliptic case in the scheme (3) or (7) or (8) given in [10] provides an additional argument to those in $[5,11]$ for considering the space of the particle as the product complex manifold determined by real-Riemannian four-dimensional manifolds $\left(\mathbb{N}_{\underline{e}}, g_{\underline{e}}\right)$ and $\left(\mathbb{N}_{\underline{n}}, g_{\underline{n}}\right)$ whose intrinsic properties express the external electromagnetic and external nuclear fields, respectively. More precisely, we have to consider a supercomplex fibre bundle generated by those manifolds with the supercomplex structure determined as in [12], but we shall leave this very formal question to a separate, more mathematical paper.

As in [1], in order to simplify further considerations, we suppose that the topologies of $\mathbb{N}_{\mathrm{e}}$ and $\mathbb{N}_{\underline{n}}$ as well as their maximal atlasses coincide, so we are going to use the single notation $\mathbb{N}$. We stick, however, to the assumption that the metrics $g_{\mathrm{e}}$ and $g_{\mathrm{n}}$ are different, in general. Let us form the complex metric

$$
g=g_{\underline{\mathrm{e}}}+i g_{\underline{\mathrm{n}}}
$$

and consider $\mathbb{N}$ a real-Riemannian four-dimensional manifold, endowed with the Riemannian complex metric $g$. We are interested in the spinor solutions on $\mathbb{M}$ of the generalised Dirac equation in the presence of 
its field $i \boldsymbol{\Gamma} / \underline{e}$ (expressed by a suitable deformation of the space of observations):

$D \psi=0, \quad \square \boldsymbol{\Gamma}=-i \underline{e} \boldsymbol{j}, \quad \operatorname{Div} \boldsymbol{\Gamma}=0, \quad \boldsymbol{\Gamma}=\boldsymbol{\Gamma}^{\mathrm{n}}-i \boldsymbol{\Gamma}^{\mathrm{e}}$.

The field is regarded as self-electromagnetic and, at the same time, as self-nuclear. The real and imaginary parts of the (local) spinor connections $\Gamma_{k}$ are interpreted (locally) as components of the nuclear and electromagnetic fields, respectively. In a local co-ordinate $\operatorname{system}\left(x^{1}, x^{2}, x^{3}, x^{0}\right)$ the general Dirac operator reads

$$
D=\gamma^{k}\left[\left(\partial / \partial x^{k}\right)+\Gamma_{k}\right]+\underline{m} I_{4} .
$$

The symbol $\boldsymbol{j}$ in (10) denotes the complex current generated by $\psi$ :

$$
j=\underline{e} \bar{\psi}^{j} \gamma_{j k} \psi^{k}, \quad \gamma_{j k}=\left(\gamma_{j k}^{1}, \gamma_{j k}^{2}, \gamma_{j k}^{3}, \gamma_{j k}^{0}\right),
$$

where $\bar{\psi}^{j}$ stands for the complex conjugate of $\psi^{j}$.

More generally, we can assume that the different parts $\boldsymbol{\Gamma}^{\mathrm{n}}$ and $i \boldsymbol{\Gamma}^{\mathrm{e}}$ of the connection $\boldsymbol{\Gamma}$ satisfy different equations. In particular, the Yang-Mills equation for $\Gamma^{\mathrm{n}}$ and the Maxwell equation for $i \boldsymbol{\Gamma}^{\mathrm{e}}$ can be attained. Let us notice, however, that we can choose the elements of metrics in such a way that the Yang-Mills equation is reduced to the Maxwell equation [13]. Thus the form introduced in (10) is of a general character and allows us to include different types of fields described by the same equation, but considered on different manifolds.

The complex-analytical method [1] of solving (10) can be summarized by the following result: Suppose that $\mathbb{N}$ is a $C^{\infty}$-differentiable four-dimensional (real-) Riemannian manifold with complex metric (9), where $g_{\mathrm{e}}$ and $g_{\mathrm{n}}$ are (real-) Riemannian. Let, further, $\psi$ : $\mathbb{N} \rightarrow \mathbb{C}^{4}$ be a $C^{\infty}$-differentiable solution of (10) with $D$ given in a local co-ordinate system $\left(x^{1}, x^{2}, x^{3}, x^{0}\right)$ by (11), where $\boldsymbol{\Gamma}=\left(\Gamma_{k}\right), k=1,2,3,0$, and $\Gamma_{k}$ are the spinor connections on $\mathbb{N}$. Then this system is equivalent to a system of the form

$$
\begin{aligned}
& {\left[a_{1}^{k}\left(\partial^{1}+i \underline{e} \Gamma_{1}^{0}\right)-(-1)^{k} a_{2}^{k}\left(\partial^{2}+i \underline{e} \Gamma_{2}^{0}\right)\right] \psi^{k}} \\
& =\left\{\begin{array}{r}
{\left[a_{3}^{1} \partial^{3}+a_{0}^{1} \partial^{0}-c^{1}(\underline{e})+\underline{m}\right] \phi^{2}+b^{1}\left(\partial^{0}+i \underline{e} \Gamma_{0}^{0}\right) \phi^{0}} \\
\text { for } k=1, \\
{\left[a_{3}^{2} \partial^{3}+a_{0}^{2} \partial^{0}+c^{1}(\underline{e})-\underline{m}\right] \phi^{1}+b^{1}\left(\partial^{0}+i \underline{e} \Gamma_{0}^{0}\right) \phi^{3}} \\
\text { for } k=2, \quad(13) \\
{\left[a_{3}^{3} \partial^{3}+a_{0}^{3} \partial^{0}+c^{1}(\underline{e})-\underline{m}\right] \phi^{0}+b^{2}\left(\partial^{0}+i \underline{e} \Gamma_{0}^{0}\right) \phi^{2}} \\
\text { for } k=3, \\
{\left[a_{3}^{0} \partial^{3}+a_{0}^{0} \partial^{0}-c^{0}(\underline{e})+\underline{m}\right] \phi^{3}+b^{2}\left(\partial^{0}+i \underline{e} \Gamma_{0}^{0}\right) \phi^{1}} \\
\text { for } k=4 ;
\end{array}\right. \\
& \square \boldsymbol{\Gamma}=-i \underline{\boldsymbol{j}} \boldsymbol{j}, \quad \operatorname{Div} \boldsymbol{\Gamma}=0,
\end{aligned}
$$

under the conditions

$$
\begin{aligned}
& a_{k}^{j}[g]=1 \quad \text { for } \quad k=1,2 \quad \text { and } b^{j}[g]=0, \\
& \Delta \Gamma^{0}=-i \underline{e} \boldsymbol{j}, \quad \operatorname{div} \Gamma^{0}=0, \quad \operatorname{div} \boldsymbol{j}^{0}=0,
\end{aligned}
$$

where the complex-valued functionals $a_{k}^{j}, b^{j}$ and $c^{j}(\underline{e})$ depend, in general, on $g$, but do not depend on the operators $\partial^{\mathrm{k}}=\partial / \partial y^{k}$ and, in addition, $a_{k}^{j}$ and $b^{j}$ do not depend on the parameters $\underline{e}$ and $\underline{m}$.

Moreover, any solution of the system can be expressed in the form

$\phi^{1}-\phi_{1}^{0}=Y_{1}\left[\phi^{2}-\phi_{0}^{2}\right], \phi^{2}-\phi_{0}^{2}=Y_{1}^{-1}\left[\phi^{1}-\phi_{0}^{1}\right]$,
$\phi^{3}-\phi_{0}^{3}=Y_{2}\left[\phi^{0}-\phi_{0}^{0}\right], \phi^{0}-\phi_{0}^{0}=Y_{2}^{-1}\left[\phi^{3}-\phi_{0}^{3}\right]$,

where

$$
\begin{array}{cr}
Y_{j}[\psi]=Y_{j}\left(y^{1}, y^{2}, y^{3}, y^{0}, \underline{e}, \underline{m}, \psi\left(y^{1}, y^{2}, y^{3}, y^{0}, \underline{e}, \underline{m}\right)\right) \\
\text { for } j=1,2 \\
\phi_{0}^{k}=\Phi^{k}\left(y^{1}+i y^{2}, \underline{e}, F\left(y^{1}, y^{2}\right)\right) & \text { for } k=1,3, \\
\phi_{0}^{k}=\Phi^{k}\left(y^{1}-i y^{2}, \underline{e}, F\left(y^{1}, y^{2}\right)\right) & \text { for } k=2,0, \\
F\left(\psi_{1}^{0}, y_{2}^{0}\right)=\int_{\mathbb{C}} K\left(y^{1}, y^{2}, y_{0}^{1}, y_{0}^{2}\right) &
\end{array}
$$$$
\cdot\left[\left(\underline{\Phi}^{1} \underline{\Phi}^{2}+\underline{\Phi}^{2} \underline{\Phi}^{1}\right)-i\left(\underline{\Phi}^{2} \underline{\Phi}^{1}-\underline{\Phi}^{1} \underline{\Phi}^{2}\right)\right] \mathrm{d} y^{1} \mathrm{~d} y^{2},
$$$$
K\left(y^{1}, y^{2}, y_{0}^{1}, y_{0}^{2}\right)=\int_{0}^{1} \log \left|t z_{0}-z\right| \mathrm{d} t
$$

$$
\begin{aligned}
= & -1+\log \left|\frac{z_{0}-z}{z}\right|-\frac{z_{0} \bar{z}+z \bar{z}_{0}}{4\left|z_{0}\right|^{2}} \log \left|\frac{z_{0}-z}{z}\right| \\
& -\frac{4\left|z_{0}\right|^{2}|z|^{2}+\left(z_{0} \bar{z}+z \bar{z}_{0}\right)^{2}}{\left|z_{0}\right|^{2}\left[4\left|z_{0}\right|^{2}|z|^{2}-\left(z_{0} \bar{z}+z \bar{z}_{0}\right)\right]^{\frac{1}{2}}}
\end{aligned}
$$

$$
\cdot \operatorname{Arctan} \frac{\left|z_{0}\right|^{2}}{\left.2\left|z_{0}\right|^{2}|z|^{2}-\left|z_{0}\right|^{2}\left(z_{0} \bar{z}+z \bar{z}_{0}\right)-z_{0} \bar{z}+z \bar{z}_{0}\right)^{2}} \text {. }
$$

The functions $\Phi=\Phi^{k}\left(y^{1}+i y^{2}, \underline{e}, \underline{\theta}\right), k=1,3$, and $\underline{\Phi}=\Phi^{k}\left(y^{1}-i y^{2}, \underline{e}, \underline{\theta}\right), k=2,4$, are the partial Fourier transforms with respect to the variable $\varepsilon$ of arbitrary four functions $\Psi^{k}: \mathbb{C}^{2} \rightarrow \mathbb{C}$, holomorphic for $k$ odd: $\underline{\Psi}^{k}=\Psi^{k}\left(y^{1}+i y^{2}, \varepsilon+i \theta\right), k=1,3$, and antiholomorphic for $k$ even: $\Psi^{k}=\Psi^{k}\left(y^{1}-i y^{2}, \varepsilon-i \theta\right), k=2,0$. The transformation $Y_{1}$ is determined by substituting the expressions (15) into (13) and, similarly, $Y_{2}$ by substituting the expressions (16) into (14).

The effectiveness of the method leading to explicit formulae is caused by the three reasons at least. Firstly, replacing the usual space-time by a curved space-time does not affect the idea of applying the Fourier transform with respect to the electric charge e treated as a variable. Secondly, canonical spinor con- 
nections $\Gamma_{k}^{0}, k=1,2$, are constructed and expressed as $G * j_{k}^{0}$, the convolution $*$ of the Green operator $G$ with the canonical currents $j_{k}^{0}$. The interpretation of this formula corresponds to the construction of the electromagnetic potential $\Gamma_{0}^{0}$ for $j_{0}^{0}=\varrho$ in the case of electron distribution with the density $\varrho$ : the 0 -component of the current is then the electron density. In such a way the potential of the self-consistent field is determined in the many-electron theories, e.g. in the theory of Hartree. Thirdly, in contrast to the usual procedure of solving the equations

$$
\begin{aligned}
& a_{k}^{j}[g]=0 \quad \text { for } \quad k=3,0 \quad \text { and } \quad c^{j}(\underline{e}, \underline{m})=0, \\
& c^{1}(\underline{e}) \phi^{2}=\underline{m} \phi^{2}, \quad c^{2}(\underline{e}) \phi^{1}=\underline{m} \phi^{1}, \\
& c^{3}(\underline{e}) \phi^{0}=\underline{m} \phi^{0}, \quad c^{0}(\underline{e}) \phi^{3}=\underline{m} \phi^{3}
\end{aligned}
$$

before (13) and (14) and then bringing back the solutions in (13) and (14), here the order is successfully reversed $[4,5,14]$.

It is clear that the system (13)-(14) can be considered a starting point to seek selection rules for curved spaces of particles corresponding to really existing particles. In such a way we have converted a hyperbolic problem concerned with the system (7)-(8) in [5] to an elliptic one concerned with the system

$$
\begin{aligned}
& a_{k}^{j}[g]=1 \quad \text { for } \quad k=1,2 \quad \text { and } b^{j}[g]=0, \\
& \Delta \boldsymbol{\Gamma}^{0}=-i \underline{\underline{e}} \boldsymbol{j}, \quad \operatorname{div} \boldsymbol{\Gamma}^{0}=0, \quad \operatorname{div} \boldsymbol{j}^{0}=0 .
\end{aligned}
$$

Such a result gives an additional information on the transformations $v_{\mathrm{e}}$ and $v_{\mathrm{n}}$ of $[2,3,11]$, describing the correspondence between the space of the particle and the space of observations. Another characterization of this correspondence has been determined in the preceding section in terms of the transformation $\mathbf{S}$ in (2). Besides, by that section the hyperbolic and elliptic problems mentioned here can be specified as SO-hyperbolic and SO-elliptic ones.
Still, in the light of the preceding section we have to study more carefully the conclusion that in the symplectic cases in the scheme (3) the Dirac equation (5) describes the Dirac field of mass $\sim 1 / R$, interacting with the electromagnetic field. To this end we have to linearize the spinor connections $\Gamma_{k}$ appearing in the Dirac operator (11).

\section{Linearization of the Spinor Connections}

Following [3], we are going to linearize the spinor connections $\Gamma_{k}$; thus we assume

$$
\gamma^{j}=\alpha_{l}^{j} \gamma_{0}^{l}, \quad \partial_{\mathbf{k}} \equiv \partial / \partial x^{k}=\beta_{k}^{l} \partial_{l}^{0},
$$

where $\gamma_{0}^{l}$ and $\partial_{l}^{0}$ correspond to the Minkowski case. We are interested in studying the effect of the local Gâteaux variation on the Minkowski metric $\eta^{j k}$ considered in the curved (pseudo-riemannian) space-time - the space of observations $\mathbb{M}$ corresponding to the space of the particle $\mathbb{N}_{e} \times \mathbb{N}_{\underline{n}}$ in the sense of [2]. Therefore the metric of $\mathbb{M}$ is real pseudo-riemannian. Without ambiguity we shall denote it in this section by $g$ and, locally, by $\left[g_{j k}\right]$ in contrast to Sect. 2, where $g$ denoted the complex-Riemannian metric of $\mathbb{N}_{\underline{e}} \times \mathbb{N}_{\underline{n}}$.

We suppose that

$$
\begin{aligned}
& \alpha_{l}^{j} \equiv \alpha_{l}^{j}(t)=\delta_{l}^{j}+t \delta \alpha_{l}^{j}+o(t), \\
& \beta_{k}^{l} \equiv \beta_{k}^{l}(t)=\delta_{k}^{l}+t \delta \beta_{k}^{l}+o(t),
\end{aligned}
$$

where $\delta_{l}^{j}$ denotes the Kronecker symbol and the supports of $\delta \alpha_{l}^{j}$ and $\delta \beta_{k}^{l}$ are compact. By (17)-(18) and the commutation rules for $\gamma^{j}$, in particular for $\gamma_{0}^{j}$, we get

$g_{l k}=\eta_{l k}+t \delta g_{l k}+o(t), \quad g^{j k}=\eta^{j k}+t \delta g^{j k}+o(t)$,

$g_{j k} I_{4}=\frac{1}{2}\left(\gamma^{j} \gamma^{k}+\gamma^{k} \gamma^{j}\right), \quad \eta_{j k} I_{4}=\frac{1}{2}\left(\gamma_{o}^{j} \gamma_{0}^{k}+\gamma_{0}^{k} \gamma_{0}^{j}\right)$.

Since $g^{j k} g_{l k}=\delta_{l}^{j}, \eta^{j k} \eta_{j l}=\delta_{l}^{j}$, we arrive at the formulae

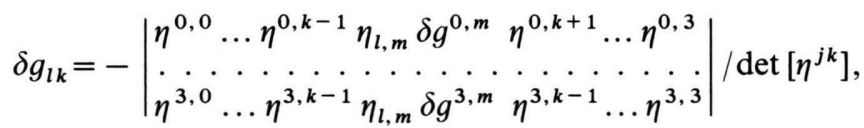

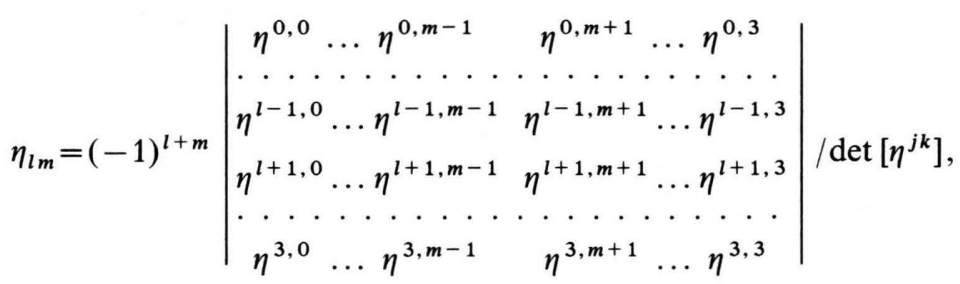

$$
\begin{aligned}
& \delta g^{j m}=\eta^{l k}\left(\delta_{l}^{j} \delta \alpha_{k}^{m}+\delta_{k}^{m} \delta \alpha_{l}^{j}\right) \equiv \eta^{j k} \delta \alpha_{k}^{m}+\eta^{l m} \delta \alpha_{l}^{j} .
\end{aligned}
$$


From (20)-(22) we infer, in particular, that the supports of $\delta g_{l k}$ and $\delta g^{j k}$ are compact (we have also $\left.\delta g_{l k}=-\eta_{j l} \eta_{m k} \delta g^{j m}\right)$.

Now, we can calculate explicitly $\delta \Gamma_{k}$; we recall that

$$
\Gamma_{k}=\frac{1}{4} \gamma^{j}\left(\partial_{k} \gamma_{i}-\gamma_{l} \Gamma_{j k}^{l}\right)-\frac{1}{32} \operatorname{Tr}\left(\gamma \gamma^{j} \partial_{k} \gamma_{j}\right) \gamma
$$

with $\gamma_{j}=g_{j k} \gamma^{k}$ and $\gamma=\frac{1}{24} \varepsilon_{j k l m} \gamma^{i} \gamma^{k} \gamma^{l} \gamma^{m}$, where $\varepsilon_{j k l m}$ denotes the totally antisymmetric Levi-Civita tensor and $\Gamma_{j k}^{l}$ the usual Christoffel symbols:

$$
g_{l m} \Gamma_{j k}^{l}=\frac{1}{2}\left(\partial_{j} g_{m k}+\partial_{k} g_{j m}-\partial_{m} g_{j k}\right) .
$$

We have

$$
\begin{aligned}
\delta\left(\gamma^{j} \partial_{k} \gamma_{j}\right)= & \delta\left[\left(\delta_{j^{\prime}}^{j}+t \delta \alpha_{j^{\prime}}^{j}\right) \eta_{0}^{j^{\prime}}\left(\delta_{k k}^{k^{\prime}}+t \delta \beta_{k}^{k^{\prime}}\right)\right] \\
& \cdot \partial_{k^{\prime}}^{0}\left[\left(\eta_{j l}+t \delta g_{j l}\right)\left(\delta_{m}^{l}+t \delta \alpha_{m}^{l}\right) \gamma_{0}^{m}\right] \\
= & \gamma_{0}^{j^{\prime}} \gamma_{0}^{m} \delta_{j^{\prime}}^{j} \delta_{k^{\prime}}^{k}\left(\delta_{m}^{l} \partial_{k^{\prime}}^{0} \delta g_{j l}+\eta_{j l} \partial_{k^{\prime}}^{0} \delta \alpha_{m}^{l}\right) \\
= & \gamma_{0}^{j^{\prime}} \delta_{j^{\prime}}^{j} \gamma_{0}^{m} \delta_{k}^{k^{\prime}}\left(\partial_{k^{\prime}}^{0} \delta g_{j m}+\eta_{j l} \partial_{k^{\prime}}^{0} \delta \alpha_{m}^{l}\right) \\
= & \gamma_{0}^{j} \gamma_{0}^{m}\left(\partial_{k}^{0} \delta g_{j m}+\delta_{j l} \partial_{k}^{0} \delta \alpha_{m}^{l}\right) .
\end{aligned}
$$

Since $\gamma_{l}=g_{l m} \gamma^{m}$, then, in analogy to (25) and, by (24), we get

$$
\begin{aligned}
\delta\left(\gamma^{j}\right. & \left.\gamma_{l} \Gamma_{j k}^{l}\right)=\frac{1}{2} \delta\left[\gamma^{j} \gamma^{m}\left(\partial_{j} g_{m k}+\partial_{k} g_{j m}-\partial_{m} g_{j k}\right)\right] \\
= & \frac{1}{2} \gamma_{0}^{j^{\prime}} \gamma_{0}^{m^{\prime}} \delta_{j^{\prime}}^{j} \delta_{m^{\prime}}^{m} \delta\left[\delta_{j}^{j^{\prime \prime}}+t \delta \beta_{j}^{j^{\prime \prime}}\right) \delta_{j^{\prime \prime}}^{0}\left(\delta_{m k}+t \delta g_{m k}\right) \\
& +\left(\delta_{k}^{k^{\prime \prime}}+t \delta \beta_{k}^{k^{\prime \prime}}\right) \delta_{k^{\prime \prime}}^{0}\left(\eta_{j m}+t \delta g_{j m}\right) \\
& \left.\quad-\left(\delta_{m}^{m^{\prime \prime}}+t \delta \beta_{m}^{m^{\prime \prime}}\right) \delta_{m^{\prime \prime}}^{0}\left(\eta_{j k}+t g_{j k}\right)\right] \\
= & \frac{1}{2} \gamma_{0}^{j^{\prime}} \delta_{j^{\prime}}^{j} \gamma_{0}^{m^{\prime}} \delta_{m^{\prime}}^{m}\left(\delta_{j}^{j^{\prime \prime}} \partial_{j^{\prime \prime}}^{0}+\partial_{k}^{0} \delta g_{j m}-\partial_{m}^{0} \delta g_{j k}\right) .
\end{aligned}
$$

Next, we observe that $\gamma=\gamma_{0}+o(1), \gamma_{0}=\frac{1}{24} \varepsilon_{j k l m}$ $\cdot \gamma_{0}^{j} \gamma_{0}^{k} \gamma_{0}^{l} \gamma_{0}^{m}$, and obtain

$$
\delta\left[\operatorname{Tr}\left(\gamma \gamma^{j} \partial_{k} \gamma_{j}\right) \gamma\right]=\operatorname{Tr}\left[\gamma_{0} \delta\left(\gamma^{j} \partial_{k} \gamma_{j}\right)\right] \gamma_{0} .
$$

By (25), further direct calculation gives

$$
\begin{aligned}
& \delta\left[\operatorname{Tr}\left(\gamma \gamma^{j} \partial_{k} \gamma_{j}\right) \gamma\right] \\
& \quad=\operatorname{Tr}\left[\gamma_{0} \gamma_{0}^{j} \gamma_{0}^{m}\left(\partial_{k}^{o} \delta g_{j m}+\eta_{j l} \partial_{k}^{0} \delta \alpha_{m}^{l}\right)\right] \gamma_{0} .
\end{aligned}
$$

From (25)-(27) we infer, by (23),

$$
\begin{aligned}
\delta \Gamma_{k}= & \frac{1}{4} \gamma_{0}^{j} \gamma_{0}^{m}\left[\partial_{k}^{0} \delta g_{j m}+\eta_{j l} \partial_{k}^{0} \delta \alpha_{m}^{l}\right) \\
& \left.-\frac{1}{2}\left(\partial_{j} \delta g_{m k}+\partial_{k} \delta g_{j m}-\partial_{m} \delta g_{j k}\right)\right] \\
& -\frac{1}{32} \operatorname{Tr}\left[\gamma_{0} \gamma_{0}^{j} \gamma_{0}^{m}\left(\partial_{k}^{0} \delta g_{j m}+\eta_{j l} \partial_{k}^{0} \delta \alpha_{m}^{l}\right)\right] \gamma_{0}
\end{aligned}
$$

or, equivalently,

$\delta \Gamma_{k}=\frac{1}{4} \gamma_{0}^{j}\left(\partial_{k}^{0} \delta_{j}-\gamma_{l}^{0} \Delta_{j k}^{l}\right)-\frac{1}{32} \operatorname{Tr}\left(\gamma_{0} \gamma_{0}^{j} \partial_{k}^{0} \delta_{j}\right) \gamma_{0}$, where, in analogy to the notation (23) and (24), we set

$\delta_{j}=\gamma_{0}^{m}\left(\delta g_{j m}+\eta_{j l} \delta \alpha_{m}^{l}\right), \quad \gamma_{0}=\frac{1}{24} \varepsilon_{j k l m} \gamma_{0}^{j} \gamma_{0}^{k} \gamma_{0}^{l} \gamma_{0}^{m}$,

$g_{l m} \Delta_{j k}^{l}=\frac{1}{2}\left(\partial_{j}^{0} \delta g_{m k}+\partial_{k}^{0} \delta g_{j m}-\partial_{m}^{0} \delta g_{j k}\right)$.

Finally, the generalised Dirac operator (11) in the curved space of observations $\mathbb{M}$ becomes

$$
D(t)=\gamma_{0}^{k} \partial_{k}^{0}+\underline{m} I_{4}+t \delta D+o(t),
$$

where

$$
\begin{aligned}
\delta D= & \delta\left[\left(\delta_{j}^{l}+t \delta \alpha_{j}^{l}\right) \gamma_{0}^{j}\left(\delta_{l}^{k}+t \delta \beta_{l}^{k}\right) \partial_{k}^{0}\right] \\
& \left.+\delta\left[\delta_{j}^{k}+t \delta \alpha_{j}^{k}\right) \gamma_{0}^{j} \Gamma_{k}\right] \\
= & \gamma_{0}^{j}\left(\delta_{l}^{k} \delta \alpha_{j}^{l}+\delta_{j}^{l} \delta \beta_{l}^{k}\right) \partial_{k}^{0}+\gamma_{0}^{j} \delta_{j}^{l} \delta_{l}^{k} \partial_{k}^{0} \delta+\delta_{j}^{k} \gamma_{0}^{j} \delta \Gamma_{k} \\
= & \gamma_{0}^{j}\left(\delta \alpha_{j}^{k}+\delta \beta_{j}^{k}\right) \partial_{k}^{0}+\gamma_{0}^{k}\left(\partial_{k}^{0} \delta+\delta \Gamma_{k}\right) .
\end{aligned}
$$

Consequently, the generalised Dirac equation in the system (10) is reduced to the system of 16 real equations with respect to the Gâteaux variations $\delta \alpha_{j}^{k}$, determined by means of the explicit relations (17)-(22) and (28)-(32). The elements of the metric are explicitly and linearly determined by the spinor connections and vice versa, thus giving a further example and progress in the research programme formulated earlier by Gaveau and two of us [15]. The resulting Diraclike equation concerns the space of observations in which the particle characterized by the mass $\underline{m} \sim 1 / R$ is situated within the electromagnetic field $A_{\mu}$ produced in a self-consistent way as the realization of the curve of the space of the particle.

The main part of the result obtained deals with the determination of the particle detected in the space of observations as a realization of the curved space-time associated with the particle. Namely, the relationship between the mass and the radius of $S_{1}$ lets us determine the mass through the correspondence between the radius and the metric of the space of the particle. According to the Kałuża-Klein theories [9], the radius of $S_{1}$ is fully determined as the constant for which the transformation $\tau^{\prime}=\tau+R \phi(x)$ in the fifth dimension remains invariant; cf. (5).

The Dirac-like equation in the space of observations has been obtained by linearizing the metric of the space of the particle with respect to the coefficients $\alpha_{l}^{j}$ in (17), transforming the Dirac matrices to the space of the corresponding Clifford matrices. Thus the spinor connections $\Gamma_{k}$ characterizing the potential of the field have been determined in linear approximation as the local Gâteaux variations $\delta \Gamma_{k}$ with the respect to $\delta \alpha_{l}^{j}$. The linear approximation, giving the 
first non-vanishing increment to the potential, is convenient from the viewpoint of physical applications by the reason of giving an explicit possibility of mutual determination of parameters in the potential and in the endomorphism $\left[\alpha_{l}^{j}\right]$ acting on the Dirac matrices. In this approximation the potential of the field is effectively determined by the metric; cf. (32). The role of the potential is, in fact, played by the increments $\delta \Gamma_{k}$ to the spinor connections given by the formulae (28). They include the parameters $\Delta_{j k}^{l}$ given by (30) which depend on the variations $\delta g_{j k}$ of the metric exactly in the same way as the Christoffel symbols $\Gamma_{j k}^{l}$ depend on the metric itself. Then the dependence of $\delta \Gamma_{k}$ on $\Delta_{j k}^{l}$ has a remarkable analogy to the dependence of $\Gamma_{k}$ on $\Gamma_{j k}^{l}$; cf. (28) and (23).

\section{The Own Field and the Demagnetizing Field of the Ferromagnet}

Till now we have discussed properties of individual elementary particles described in their own spaces (of the particles) with a strictly determined metric (9); cf. [2], formula (1.2). The metric was responsible for interactions with the external field. Now we turn our attention to the construction of an equation for a system of mutually interacting particles. The most typical example concerning collections of elementary particles appearing in nature are the atomic nuclei as sets of nucleons and also the atoms, molecules or systems of atoms forming crystals or - more generally - systems of condensed phase in which sets of electrons appear.

From the viewpoint of elementary particles these sets of electrons may be treated as sets of mutually interacting electrons, submerged in the exterior field of suitably distributed nuclei. In the most general formulation the electrons have to be treated as sets of mutually interacting electrons which interact with the nucleons of each atomic nucleus. In the latter case all the interactions are expressed by interactions between elementary particles: electron-electron, electron-nucleon, and nucleon-nucleon, where the individual properties of the particles are described by the equations corresponding to these particles.

The simplest case is provided by an atomic nucleus consisting of mutually interacting nucleons. Other simple examples are constituted by crystals which are considered collections of electrons interacting in the field of atomic nuclei treated as the point sources of charge. The last case, including the electron-nucleon interactions, is more complicated but the formulation of the problem, described below, can easily be extended to the general case without any extra assumptions; only with some complication of the resulting formulae. Thereafter, we concentrate on an arbitrary many-electron system in an exterior field. Independently of the fact whether we are dealing with a thin film or a bulk with an interior atom together with its nearest neighbours or with an adatom with its nearest neighbours restricted by a surface, we are interested in a system of $k+1$ electrons. If these electrons belong to $n$ atoms, we have a decomposition $k+1=k_{1}+\ldots+k_{n}$.

Then the Dirac equation in the system (1) becomes a Breit equation [16] extended for $k+1$ electrons so that

$$
\begin{aligned}
D_{0}= & H-E, \quad H=\sum_{j=1}^{k+1} H^{j}+(\hbar \underline{e} c)^{2} \\
& \cdot \sum_{j \neq j^{\prime}} \frac{1}{r_{j j^{\prime}}}\left[I_{v}-\frac{1}{2} \boldsymbol{a}^{j} \boldsymbol{a}^{j^{\prime}}+\frac{1}{2 r_{j j^{\prime}}^{2}}\left(\boldsymbol{a}^{j} \boldsymbol{r}_{j j^{\prime}}\right)\left(\boldsymbol{a}^{j^{\prime}} \boldsymbol{r}_{j j^{\prime}}\right)\right] .
\end{aligned}
$$

Here $E$ denotes the total energy and $H$ the total hamiltonian. $H$ is the sum of the hamiltonians $H^{q}, q=1, \ldots$, $k+1$, of individual electrons and the interaction term $H_{\text {int }}$ which includes the demagnetizing term

$$
H_{\mathrm{dem}}=\frac{1}{2}(\hbar \underline{e} c)^{2} \sum_{q \neq q^{\prime}} \frac{1}{r_{q q^{\prime}}^{3}}\left(\alpha^{q} \boldsymbol{r}_{q q^{\prime}}\right)\left(\alpha^{q^{\prime}} \boldsymbol{r}_{q q^{\prime}}\right),
$$

where $\boldsymbol{r}_{q q^{\prime}}$ stand for the euclidean vectors between the $q$ th and $q^{\prime}$ th electrons, and $r_{q q}$, for their euclidean distance. Similarly, $\boldsymbol{\alpha}^{q}$ denotes the $q$-dependent coefficient matrix $\left[\alpha_{l}^{j}\right]$ for the $q$-dependent generators $\gamma^{q j}$ of the Clifford algebra corresponding to the $q$ th electron, as indicated in (17), chosen in the imaginary Majorana representation. Of course, $v=2^{4 k+3}$.

Now, each $H^{q}$ can be expressed as

$$
\begin{array}{r}
H^{q}=\left(\gamma^{q 0}\right)^{-1} D^{q}, \quad D^{q}=D_{0}^{q}+D_{1}^{q}-i \underline{e} \gamma^{q 0} A_{0}^{q}, \\
q=1, \ldots, k+1,
\end{array}
$$

where $\gamma^{q 0}$ is the vector whose components are 0 -components of the generators of the Clifford algebras corresponding to the $q$ th electron: $\gamma^{q 0}=I_{v}, v=2^{4 k+3}$, or $\gamma^{q 0}=-i \gamma^{q p}$ (cf. (12) in [17], while

$D_{0}^{q}=\gamma^{q 0}\left[\left(\partial / \partial x_{q}^{0}\right)+i \underline{e} A_{0}^{q}\right]$,

$D_{1}^{q}=\gamma^{q l}\left[\left(\partial / \partial x_{q}^{l}\right)+i \underline{e} A_{l}^{q}\right]+I_{v} \underline{m}, \quad l=1, \ldots, p-1$.

We are going to reduce, according to the scheme (7), the system of $k+1$ interacting electrons coming from 
the $n$ atoms in question to the system of $k+1$ free electrons considered individually in the external $2^{4 k+3}$-dimensional field caused by the remaining electrons as described by the Dirac-Maxwell system of the type (11):

$$
\tilde{D}^{Q} \tilde{\Psi}^{q}=0, \quad \square_{p} \Gamma^{q}=-i \underline{\underline{e}} j^{q}, \quad \operatorname{Div}_{p} \Gamma^{q}=0,
$$

where $p=8 k+5$,

with the usual meaning of $\square=\square_{p}$ and $\operatorname{Div}=\operatorname{Div}_{p}$ in $S, \operatorname{dim} S=p, S$ containing the domain of definition of $\tilde{\Psi}^{q}$, and $\Gamma^{q}=i \underline{e} \boldsymbol{A}^{q}$.

Precisely, according to [10], the SO-hyperbolic case in the scheme (7) is described by a pseudo-riemannian Hurwitz pair $\left(\mathbf{B}_{\mathrm{M}}, V\right)$ with metric

$\left[g_{a b}(z)\right]$

$$
=\left[\begin{array}{cc}
{\left[g^{0}{ }_{\mu v}(x)+\underline{e}^{2} A_{\mu} A_{v}\right]} & {\left[\underline{e} A_{\mu}^{\alpha}(x) K_{\alpha}^{s}(y) g_{s t}^{0}(y)\right]} \\
{\left[\underline{e} A_{v}^{\alpha}(x) K_{\alpha}^{t}(y) g_{s t}^{0}(y)\right]} & {\left[g_{s t}^{0}(y)\right]}
\end{array}\right],
$$

where

$$
\left[g_{a b}^{0}(z)\right]=\left[\begin{array}{cc}
{\left[g_{\mu v}^{0}(x)^{0}\right.} & 0 \\
0 & {\left[g_{s t}^{0}(y)\right]}
\end{array}\right], \quad \begin{aligned}
& 0 \text { being the zero } \\
& 4 \times(p-4) \text {-matrix }
\end{aligned}
$$

$A_{\mu}^{\alpha}(x)$ are gauge fields,

$$
\begin{aligned}
& A_{\mu} A_{v}:=A_{\mu}^{\alpha}(x) A_{v}^{\beta}(x) K_{\alpha}^{s}(y) K_{\beta}^{t}(y) g_{s t}^{0}(y) ; \\
& x=\left(x^{\mu} ; \mu=0, \ldots, 3\right), \quad y=\left(y^{s} ; s=1, \ldots, p-4\right), \\
& z=\left(z^{a} ; a=1, \ldots, p\right)
\end{aligned}
$$

denote local co-ordinate systems in $\mathbb{M}_{0}, \mathbb{M}_{\#}, \mathbb{M}$, respectively, $\mathbb{M}=\mathbb{M}_{0} \times \mathbb{M}_{\#}, \mathbf{B}_{\mathbf{M}}$ is a fibre bundle with base space $\mathbb{M}_{0}$ and quotient typical fibre $\mathbb{M}_{\#}$ of a Lie group $G$ and its subgroup $G_{0}$, while $K_{\alpha}^{s}(y)$ are the Killing vectors connected with the transformations $y^{s} \mapsto y^{s}+\varepsilon^{\alpha}(x) K_{\alpha}^{s}(y)$ of the group $G$ treated as the isometry groups of $\mathbb{M}_{\#},\left(\varepsilon^{\alpha}(\xi)\right)$ being an orthonormal basis of the tangent space $S=T_{\xi} \mathbb{M}_{0}$ at a point $\xi$. If $\left(\varepsilon^{\alpha}(\zeta)\right)$ is an orthonormal basis of $T_{\zeta} \mathbb{M}$ at $\zeta$ and $\left(e_{i}\right)$ an orthonormal basis of the $2^{4 k+3}$-dimensional real vector space $V$ containing the ranges of all $\widetilde{\Psi}^{q}$ then, according to the scheme (7), the scalar products $(,)_{T_{z} \mathrm{M}}$ and $(,)_{V}$ have to satisfy the relations

and

$$
\left[\left(\varepsilon^{\alpha}(z), \varepsilon^{\beta}(z)\right)_{T_{x} \mathrm{M}}\right]=\left[\begin{array}{cc}
I_{8 k+4} & 0 \\
0^{T} & 1
\end{array}\right]
$$

$$
\left[\left(e_{r}, e_{s}\right)_{V}\right]=\left[\begin{array}{rr}
I_{\frac{1}{2} v} & 0_{\frac{1}{2} v} \\
O_{\frac{1}{2} v} & -I_{\frac{1}{2} v}
\end{array}\right] \text {, }
$$

where $v=2^{4 k+3}$ and 0 denotes the zero $1 \times(8 k+4)$ matrix. The metric (4) is an example of $\left[g_{a b}(z)\right]$.

Now let $\Omega \subset T_{z} \mathbb{M}$ and $f \in V$. Suppose that $\Omega$ contains with every $\underline{a}$ its inverse $\underline{a}^{-1}$ provided that $(\underline{a}, \underline{a})_{T_{z} \mathrm{M}} \neq 0$. Under the $\Omega$-ray generated by $f$ in $T_{z} \mathbb{M}$ we mean the set of all vectors in $T_{z} \mathbb{M}$ obtained by successive multiplication of $f$ by elements of $\Omega$, i.e. vectors of the form $\omega f, \omega_{2}\left(\omega_{1} f\right)$ etc., where $\omega, \omega_{1}, \omega_{2}$ etc. belong to $\Omega$. In particular, we may consider a $T_{z} \mathbb{M}$-ray $V_{0}$ generated by $f$ in $V: V_{0}=\left(T_{z} \mathbb{M}\right) f$. Arguing as in [12] (Lemma 8) we conclude that $V$ is a finite direct sum

$$
\begin{gathered}
V=\bigoplus_{q=1}^{2^{m}} V_{q}, \quad \text { where } \quad \operatorname{dim} V_{q}=2^{4 k+3-m} \geqq 8 \\
\text { and } \quad 2^{m}>k+1,
\end{gathered}
$$

of mutually isomorphic real vector subspaces $V_{q}$, independent of $z$ and invariant under the action of $T_{z} \mathbb{M}$ as being $T_{z} \mathbb{M}$-rays in $V$. Moreover, in each $V_{q}$ the multiplication scheme

$$
\varepsilon^{\alpha}(z) e_{s}^{q}=i\left(\gamma^{q \alpha}\right)_{s}^{t}(z) e_{t}^{q}, \alpha=1, \ldots, p ; s, t=1, \ldots, v,
$$

where $\left(e^{q \alpha}\right)$ are some orthonormal bases of $V_{q}$, respectively, determines uniquely the product $\underline{a} g$ for $g \in V_{q}$ and $\underline{a} \in T_{z} \mathbb{M}$.

With such a choice of the $q$-dependent generators $\gamma^{q \alpha}$ of the Clifford algebra corresponding to the $q$ th electron, the block decomposition (35) of the space $V$ yields the decomposition

$$
\Psi=\left[\begin{array}{cccc}
\Psi^{1} & 0 & \ldots & 0 \\
0 & \Psi^{2} & \ldots & 0 \\
\ldots & \ldots & \ldots & \ldots \\
0 & 0 & \ldots & \Psi^{2 m}
\end{array}\right]=\sum_{q=1}^{2^{m}} \tilde{\Psi}^{q}-2^{m} I_{2^{4 k+3}}
$$

with

$$
\tilde{\Psi}^{q}=\left[\begin{array}{lll}
I^{1} & 0_{1} & 0_{2} \\
0_{1}^{T} & \Psi^{q} & 0_{3} \\
0_{2}^{T} & 0_{3}^{T} & I_{3}
\end{array}\right], \begin{aligned}
& I^{1}=I_{2^{4 k+3-m}(q-1)}, \\
& I^{2}=I_{2^{4 k+3-m}\left(2^{m}-q\right)},
\end{aligned}
$$

$\left(0, O_{1}, O_{2}, O_{3}\right)$ denoting the corresponding zero matrices of the spinor $\Psi$ involved (i.e. such that $D \Psi=0$, $\square_{p} \Gamma=0$, and $\operatorname{Div}_{p} \Gamma=0$ ) so that the associated DiracMaxwell system is really of the form (33) with e.g.

$$
\begin{aligned}
\tilde{D}^{q} & =D^{q}+\frac{2}{\hbar c}\left(\gamma^{q 0}+I_{v}\right)^{-1} H_{\mathrm{int}}^{q}, \quad q \leqq k+1, \\
\tilde{D}^{q} & =\gamma^{q 0} \sum_{j \in\left\{l_{q}\right\}}\left(\gamma^{j 0}\right)^{-1} D^{j}+\frac{2}{\hbar c}\left(\gamma^{q 0}+I_{v}\right)^{-1} \sum_{j=1}^{2^{m}} H_{\mathrm{int}}^{j}, \\
q & >k+1
\end{aligned}
$$


where

$$
\begin{aligned}
& H_{\mathrm{int}}^{q}=(\hbar \underline{e} c)^{2} \sum_{j \neq q} \frac{1}{r_{j q}}\left(I_{v}-\frac{1}{2} \alpha^{j} \alpha^{q}\right)+H_{\mathrm{dem}}^{q}, \\
& H_{\mathrm{dem}}^{q}=\frac{1}{2}(\hbar \underline{e} c)^{2} \sum_{j \neq q} \frac{1}{r_{q j}^{3}}\left(\boldsymbol{\alpha}^{q} \boldsymbol{r}_{q j}\right)\left(\boldsymbol{\alpha}^{j} \boldsymbol{r}_{q j}\right),
\end{aligned}
$$

$\left\{l_{q}\right\}$ denotes the set (consisting of at least two elements) of those $j$ for which $\left(\gamma^{j 0}\right)^{-1} D^{j}$ depend on exactly $l_{q}$ degrees of freedom among $4(k+1)+1$ possible. The total number of possibilities is

$$
\frac{1}{8} \sum_{l=0}^{4 k+3}\left(\begin{array}{c}
4 k+3 \\
l
\end{array}\right)=\frac{1}{8} \cdot 2^{4 k+3}=2^{4 k}
$$

and hence, by (35), we conclude that $m=4 k$.

In order to have a full generality, we need to pass from the collection of tangent spaces $T_{z} \mathbb{M}$, i.e. from the tangent bundle $T \mathbb{M}$, to the manifold $\mathbb{M}$ (the space of observations) itself. In terms of the tetrad formalism, if $x, y, z$ are as in the formulae (34) and $\zeta\left(z ; z_{0}\right)$ $=\left(\zeta^{\alpha}\right)\left(z ; z_{0}\right)$ are local co-ordinates in $\mathbb{M}$ around a point $z_{0}$ (interpreted as inertial ones), then

$$
\left(\partial / \partial \zeta^{\alpha}\right) \lambda_{a}^{\alpha}=\partial / \partial z^{a}, \quad \alpha, a=1, \ldots, p,
$$

where $\lambda$ is the field of tetrads:

$$
\lambda_{a}^{\alpha}\left(z ; z_{0}\right)=\left[\begin{array}{cc}
{\left[\lambda_{\mu}^{m}\left(x ; z_{0}\right)\right]} & {\left[A_{\mu}^{\beta}\left(x ; z_{0}\right) K_{\beta}^{j}\left(y ; z_{0}\right)\right]} \\
0^{T} & {\left[\lambda_{l}^{l}\left(y ; z_{0}\right)\right]}
\end{array}\right],
$$

and, as before, 0 stands for the zero $4 \times(p-4)$-matrix. The base vectors $\varepsilon^{\alpha}\left(z_{0}\right)$ have to be replaced by $\varepsilon^{\alpha}\left(z ; z_{0}\right)$ according to the multiplication scheme

$$
\varepsilon^{\alpha}\left(z ; z_{0}\right) e_{s}^{q}=i\left(\gamma^{q a}\right)_{s}^{t}\left(z ; z_{0}\right) e_{t}^{q}, \quad \begin{aligned}
a & =1, \ldots, p ; \\
s, t & =1, \ldots, v,
\end{aligned}
$$

where

$$
\gamma^{q a}\left(z ; z_{0}\right)=\lambda_{\alpha}^{a}\left(z ; z_{0}\right) \gamma^{q \alpha}\left(z_{0}\right) .
$$

Similarly, for the covariant derivative $\nabla_{\alpha}$, we have

$$
\nabla_{\alpha}=\lambda_{\alpha}^{a}\left(\partial_{a}+\Gamma_{a}\right), \quad \Gamma_{a}=\frac{1}{2} \Sigma^{\alpha \beta} \lambda_{\alpha}^{a^{\prime}} \lambda_{\beta}^{b} g_{b b^{\prime}} \Gamma_{a a^{\prime}}^{b^{\prime}},
$$

where $\Gamma_{b_{a^{\prime}}}^{b^{\prime}}$ denote the generalised Christoffel symbols, in terms of a local co-ordinate system (34), given by the formulae $[10,18]$

$g_{b b^{\prime}}, \Gamma_{a a^{\prime}}^{b^{\prime}}=\lambda_{a^{\prime}}^{\beta}\left[\partial_{a} \lambda_{\beta b}-\frac{1}{2} \lambda_{\beta}^{b^{\prime}}\left(\partial_{a} g_{b b^{\prime}}+\partial_{b^{\prime}} g_{a b}-\partial_{b} g_{a b^{\prime}}\right)\right]$.

The coefficients $\Sigma^{\alpha \beta}$ are the generators of the semisimple Lie group $\mathrm{SO}(r+1, s)$ in the representation $D[\Lambda]$ :

$$
\begin{aligned}
& \Sigma^{\alpha \beta}=(1 / 4 i)\left[\gamma^{\alpha}, \gamma^{\beta}\right] \text { for } \quad \alpha, \beta \neq r+1, \\
& \Sigma^{\alpha, r+1}=-\Sigma^{r+1, \alpha}=\gamma^{\alpha} ; \quad \gamma^{\alpha} \neq \gamma^{q \alpha} \quad \text { (in general), }
\end{aligned}
$$

where $\Lambda$ is defined by $\zeta^{\prime}\left(z ; z_{0}\right)=\Lambda\left(z ; z_{0}\right) \zeta\left(z ; z_{0}\right)$. Since all the geometrical objects involved are well defined globally, thereafter we shall skip in notion the dependence on $z_{0}$.

We are going to derive from the system of first order differential equations (33) with the operators (36) a system of second order differential equtions which contains the Clifford matrices $\gamma^{q a}=\gamma^{q a}(z), z$ and $a$ being, as in (34), only in terms which vanish in the absence of an electromagnetic field. To this end we multiply, for each $q \leqq k+1$, both sides of the first system in (33) by the operator

$$
\bar{D}^{q}=\bar{D}_{0}^{q}+\bar{D}_{1}^{q}-i \underline{e} \gamma^{q 0} A^{q 0},
$$

where

$$
\bar{D}_{0}^{q}=-D_{0}^{q} \quad \text { and } \quad \bar{D}_{1}^{q}=-D_{1}^{q}+2 I_{v} \underline{m} .
$$

Since $\left\{\gamma^{q a}, \gamma^{q a}\right\}=2 I_{v} g^{a a^{\prime}}$, the system obtained, after multiplying through by $\hbar c / 2 \underline{m}$, becomes

$$
\begin{aligned}
& \left\{-I_{v} g^{00} \tilde{W}+\frac{\hbar c}{2 \underline{m}}\left[\partial_{l} i \gamma^{l}-\partial_{l}\left(i \gamma^{l}\right)\right] i \gamma^{l^{\prime}} \partial_{l^{\prime}}\right. \\
& -\frac{I_{v} g^{00}}{2 \hbar \underline{m} c} \tilde{W}^{2}+\frac{1}{2} \hbar \underline{m} c I_{v}\left(1-g^{00}\right) \\
& +i \frac{\hbar \underline{e} c}{2 \underline{m}}\left(-I_{v}\right) g^{a a^{\prime}} \partial_{a}\left(A_{a^{\prime}}\right) \\
& +i \frac{\hbar \underline{e} c}{2 \underline{m}} i \gamma^{l}\left(i \gamma^{l^{\prime}} A^{l^{\prime}} \partial_{l}+A_{l^{\prime}} \partial_{l} i \gamma^{l^{\prime}}\right) \\
& -\frac{\hbar \underline{e}^{2} c}{2 \underline{m}}\left(-I_{v}\right) g^{l l} A_{l}^{2} \\
& -\frac{h \underline{e} c}{2 \underline{m}} \cdot \frac{1}{2} i \sum_{l \neq l^{\prime}} \gamma^{l} \gamma^{l^{\prime}}\left(F_{l l^{\prime}}+2 i \underline{\underline{e}} A_{l} A_{l^{\prime}}\right) \\
& +i \frac{\hbar \underline{e} c}{2 \underline{m}} \cdot i \gamma^{l} \gamma^{0}\left(i F_{l 0}-\underline{e} \phi A_{l}\right)+H_{\mathrm{int}} \\
& -\frac{1}{2 \underline{m}} i \gamma^{l} i \gamma^{0} W \partial_{l}-\frac{1}{2 \underline{m}} i \gamma^{0}\left(i \gamma^{0}\right)(\tilde{W}+\hbar \underline{m} c) \\
& -\frac{1}{2 \underline{m}}\left[\partial_{l}\left(i \gamma^{l} i \gamma^{0}\right)+2 \underline{e} i \gamma^{0} \gamma^{l} A_{l}\right] W \\
& +\frac{1}{2 \underline{m}}\left(I_{v} g^{00} \partial_{0}^{2}-i \gamma^{l} i \gamma^{0} \partial_{l}\right)(W) \\
& +\frac{\hbar c}{2 \underline{m}}\left[\partial_{0}^{2} i \gamma^{0} i \gamma^{l}-\partial_{0}\left(i \gamma^{0}\right) i \gamma^{l}-2 \underline{e} i \gamma^{l} i \gamma^{0} \phi\right] \partial_{l} \\
& +\frac{1}{2} \hbar c \partial_{l}\left(i \gamma^{l}\right) i \gamma^{0}-i \hbar \underline{e} c i \gamma^{0} i \gamma^{l} A_{l}
\end{aligned}
$$




$$
\begin{aligned}
& -\frac{\hbar \underline{e} c}{2 \underline{m}} i \gamma^{l} \phi \partial_{l}\left(i \gamma^{0}\right)+i \frac{\hbar \underline{e} c}{2 \underline{m}} i \gamma^{0} A_{l} \partial_{0}\left(i \gamma^{l}\right) \\
& +\frac{1}{\hbar \underline{m} c} \gamma^{0} \hat{H}_{\mathrm{int}} \mathrm{W}-\frac{1}{\underline{m}} \gamma^{l} \hat{H}_{\mathrm{int}} \partial_{1}-\frac{1}{\underline{m}} \gamma^{a} \partial_{a}\left(\hat{H}_{\mathrm{int}}\right) \\
& \left.-i \frac{\underline{e}}{\underline{m}}\left(\gamma^{l} A_{l}+\gamma^{0} i \phi\right) \hat{H}_{\mathrm{int}}\right\} \tilde{\Psi}=0
\end{aligned}
$$

where $\tilde{W}, W, \gamma^{l}, \partial_{l}, A_{l}, A_{l}^{2}, \phi, F_{l l^{\prime}}, H_{\mathrm{int}}, \hat{H}_{\mathrm{int}}$, and $\tilde{\Psi}$ stand for $\tilde{W}^{q}, W_{q}, \gamma^{q l}, \partial_{l}^{q}, A_{l}^{q},\left(A_{l}^{q}\right)^{2}$, etc., respectively, $l, l^{\prime}$ and $a, a^{\prime}$ range from 1 to $p-1$ and $p$, respectively, $\tilde{W}^{q}=W^{q}+\hbar \underline{e} c \phi^{q}, W^{q}$ is the nonrelativistic energy of the $q$ th particle: $W^{q}=E^{q}-\hbar \underline{m} c, E^{q} \Psi^{q}=\hbar c \partial_{0}^{q} \Psi^{q}, \phi^{q}=$ $-i A_{0}^{q}$ is its scalar potential, $\left[F_{a a^{\prime}}^{q}\right]=\left[\partial_{a}^{q}\left(A_{a^{\prime}}^{q}\right)-\partial_{a^{\prime}}^{q}\left(A_{a}^{q}\right)\right]$ denotes the tensor of the electromagnetic field, and $\hat{H}_{\text {int }}^{q}=\left(\gamma^{q 0}+I_{v}\right)^{-1} H_{\text {int }}^{q}$.

The brackets ( ) in the expression $\partial_{l}\left(i \gamma^{l}\right) \tilde{\Psi}$ etc. indicate that the operator $\partial_{l}$ applies to $i \gamma^{l}$ only. The condition $\operatorname{Div}_{p} \Gamma^{q}=0$ in (33) with $\Gamma^{q}=i \underline{e} A^{q}$ gives

$$
g^{\mathrm{a} \mathrm{a}^{\prime}} \partial_{a}^{q}\left(A_{a^{\prime}}^{q}\right)+g^{a a^{\prime}} \Gamma_{a a^{\prime}}^{b^{\prime}} A_{b^{\prime}}^{q}=0,
$$

where $\Gamma_{b a^{\prime}}^{b^{\prime}}$ denote the generalised Christoffel symbols, locally determined by (38) and (39). Besides, thereafter we suppose the system to be stationary:

$\left(I_{v} g^{00} \partial_{0}^{q}-i \gamma^{q l} i \gamma^{q 0} \partial_{l}^{q}\right)\left(W^{q}\right)=0, \quad q \leqq k+1$.

The global formulation of (40) will be discussed in a subsequent paper.

\section{Dependence of the Band Structure on Temperature, Oguchi's Theorem, and the Autocorrelation Time}

Finally, we are going to show that the relativistic approach to the many-electron problem as a generalisation of the Breit equation given in Sect. 4, leads to the hamiltonian including interaction of higher orders with respect to the operators of annihilation and creation of electrons $[3,10]$. This fact will indicate the dependence of the band structure on temperature, provide us with a proof of the theorem (following the theorem of Oguchi [19]) on the linear dependence of energy of the hyperfine interaction on the values of electron and nuclear spins, and lead to more precise formulae determining the autocorrelation time [20,6].

The tensor $\left[F_{a a^{\prime}}^{q}\right]$ of the electromagnetic field gives rise to the vector $\boldsymbol{E}^{q} \equiv\left(E_{l}^{q}\right)=\left(i F_{l 0}^{q}\right)$ which has to be interpreted as the intensity of the self-electric field of the $q$ th electron and $\alpha^{q l}=i \gamma^{q l} i \gamma^{\gamma 0}$ are counterparts of the Dirac $\alpha$-matrices. Similarly, the diagonalization

$$
\sigma^{q l} \underline{H}_{l}^{q}=\frac{1}{2} i \sum_{l \neq l^{\prime}}\left(\gamma^{q l} \gamma^{q l^{\prime}}\right) F_{l l^{\prime}}^{q},
$$

where $\sigma^{q l}$ are quadratic forms of $\left(\gamma^{q l^{\prime}}\right)$, leads to the intensity $\boldsymbol{H}^{q} \equiv\left(\underline{H}_{l}^{q}\right)$ of the self-magnetic field of the $q$ th electron and to its reduced spin vector $\sigma^{q}=\left(\sigma^{q l}\right)$. Then, by (41) and (42), the system (40) can be rewritten as

$$
\begin{aligned}
-I_{v} g^{00} W= & H_{0}-H_{\mathrm{int}}+b^{l} \hat{H}_{\mathrm{int}} \partial_{l}+b_{1}\left(\hat{H}_{\mathrm{int}}\right)+b_{2} \hat{H}_{\mathrm{int}} \\
& +\varepsilon_{0}^{l} W \partial_{l}+\left(\varepsilon_{1}+\varepsilon_{2} \hat{H}_{\mathrm{int}}\right) W+\varepsilon_{0}^{p} W^{2},
\end{aligned}
$$

where

$$
\begin{aligned}
& H_{0}=\hbar \underline{e} c I_{v} g^{00} \phi-\frac{\hbar c}{2 \underline{m}}\left[\partial_{l} i \gamma^{l}-\partial_{l}\left(i \gamma^{l}\right)\right] i \gamma^{l^{\prime}} \partial_{l^{\prime}} \\
& +\frac{\hbar \underline{e}^{2} c}{2 m} I_{v} g^{00} \phi^{2}-\frac{1}{2} \hbar \underline{m} c I_{v}\left(1-g^{00}\right) \\
& -i \frac{\hbar \underline{e} c}{2 m} I_{v} g^{a a^{\prime}} \Gamma_{a a^{\prime}}^{b} A_{b} \\
& -i \frac{\hbar \underline{\underline{e}} c}{2 m} i \gamma^{l}\left(i \gamma^{l^{\prime}} A_{l^{\prime}} \partial_{l}+A_{l^{\prime}} \partial_{l} i \gamma^{l^{\prime}}\right)
\end{aligned}
$$

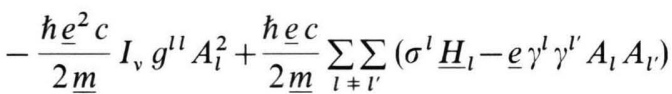

$$
\begin{aligned}
& -i \frac{\hbar \underline{e} c}{2 m}\left(\alpha^{l} \underline{E}_{l}-\underline{e} \gamma^{l} \gamma^{0} \phi A_{l}\right)+\frac{1}{2} \hbar c i \gamma^{0} \partial_{0}\left(i \gamma^{0}\right) \\
& -\frac{\hbar c}{2 \underline{m}}\left[\partial_{0} i \gamma^{0} i \gamma^{l}-\partial_{0}\left(i \gamma^{0}\right) i \gamma^{l}-2 \underline{e} i \gamma^{l} i \gamma^{0} \phi\right] \partial_{l} \\
& -\frac{1}{2} \hbar c \partial_{l}\left(i \gamma^{l}\right) i \gamma^{0}+i \hbar \underline{e} c i \gamma^{0} i \gamma^{l} A_{l} \\
& +\frac{\hbar \underline{e} c}{2 m} i \gamma^{l} \phi \partial_{l}\left(i \gamma^{0}\right)-i \frac{\hbar \underline{e} c}{2 m} i \gamma^{0} A_{l} \partial_{0}\left(i \gamma^{l}\right), \\
& b_{l}=-\frac{1}{\underline{m}} \gamma^{l}, \quad b_{1}=\frac{1}{\underline{m}} \gamma^{a} \partial_{a}, \quad b_{2}=i \frac{\underline{e}}{\underline{m}}\left(\gamma^{l} A_{l}+\gamma^{0} i \phi\right), \\
& \varepsilon_{0}^{l}=\frac{1}{2 \underline{m}} i \gamma^{l} i \gamma^{0}, \\
& \varepsilon_{1}=\frac{\underline{e}}{\underline{m}} I_{v} g^{00} \phi+\frac{1}{2} \hbar c i \gamma^{0} \partial_{0}\left(i \gamma^{0}\right) \\
& +\frac{1}{2 m}\left[\partial_{l}\left(i \gamma^{l} i \gamma^{0}\right)+2 \underline{e} i \gamma^{0} \gamma^{l} A_{l}\right], \\
& \varepsilon_{2}=(-1 / \hbar \underline{m} c) \gamma^{0}, \quad \varepsilon_{0}^{p}=I_{v} g^{00} / 2 \hbar \underline{m} c,
\end{aligned}
$$


where $W, H_{0}, H_{\mathrm{int}}, \hat{H}_{\mathrm{int}}, b_{0}^{l}, b_{1}^{0}, b_{2}^{0}, \varepsilon_{0}^{l}, \varepsilon_{1}, \varepsilon_{2}, \gamma^{l}, \delta_{l}, A_{l}$, $\phi, F_{l l^{\prime}}, \underline{E}_{l}, \underline{H}_{l}, \alpha^{l}$, and $\sigma^{l}$ stand for $W^{q}, H_{0}^{q}, \ldots, b_{0}^{q l}$, etc., respectively.

In a more concise form the system of (44) reads

$$
W=P(W) \equiv H_{1}+\varepsilon^{0} W+\varepsilon^{l} W \partial_{l}+\varepsilon^{p} W^{2},
$$

where, as before, $W \equiv W^{q}, W^{2} \equiv\left(W^{q}\right)^{2}, P \equiv P^{q}$, etc., except for $\varepsilon^{p}$ which does not depend on $q$, and

$\left.H_{1}=\left(-g^{00}\right)^{-1}\left[H_{0}-H_{\text {int }}+b^{l} \hat{H}_{\text {int }} \partial_{l}+b_{1} \hat{H}_{\text {int }}\right)+b_{2} \hat{H}_{\text {int }}\right]$,

$\varepsilon^{0}=\left(-g^{00}\right)^{-1}\left(\varepsilon_{1}+\varepsilon_{2} \hat{H}_{\text {int }}\right)$,

$\varepsilon^{l}=\left(-g^{00}\right)^{-1} \varepsilon_{0}^{l}, \quad \varepsilon^{p}=\left(-g^{00}\right)^{-1} \varepsilon_{0}^{p}$.

Consider the operator $P$. We are going to find the approximate solution to the system (45) by applying the iteration $W_{1}=P^{2}(W) \equiv P \circ P(W) \equiv P(P(W))$, $W_{2}=P^{3}(W) \equiv P \circ P \circ P(W)$, etc., where $W_{j}=W_{j}^{q}$, with the exactness to $o\left(\varepsilon^{2}\right), \varepsilon=\left[\left(\varepsilon^{0}\right)^{2}+\ldots+\left(\varepsilon^{p}\right)^{2}\right]^{\frac{1}{2}}$.

It is clear that to obtain the result calculated with the exactness specified above and independent of the unknown nonrelativistic energy $W^{q}$, it suffices to make two steps, calculating $W_{1}^{q}$ and $W_{2}^{q}$. Indeed, we get

$$
\begin{aligned}
W_{1}= & \left(I_{v}+\varepsilon^{0}+\varepsilon^{p} H_{1}\right) H_{1}+\varepsilon^{l} H_{1} \partial_{l}+\left(\varepsilon^{0}\right)^{2} W \\
& +\left(\varepsilon^{0} \varepsilon^{l}+\varepsilon^{l} \varepsilon^{0}\right) W \partial_{l}+\varepsilon^{l} \varepsilon^{l^{\prime}} W \partial_{l^{\prime}} \partial_{l}+\varepsilon^{0} \varepsilon^{p} W^{2} \\
& +\varepsilon^{p} H_{1} \varepsilon^{0} W+\varepsilon^{p} \varepsilon^{0} W H_{1}+\varepsilon^{l} \varepsilon^{0} W^{2} \partial_{l} \\
& +\varepsilon^{p} H_{1} \varepsilon^{l} W \partial_{l}+\varepsilon^{p} \varepsilon^{l} W \partial_{l} H_{1} \\
& +\varepsilon^{p}\left(H_{1} \varepsilon^{p}+\varepsilon^{p} H_{1}\right) W^{2}+o\left(\varepsilon^{2}\right)
\end{aligned}
$$

and

$$
\begin{aligned}
W_{2}= & {\left[I_{v}+\varepsilon^{0}+\left(\varepsilon^{0}\right)^{2}\right] H_{1}+\left(\varepsilon^{l}+\varepsilon^{0} \varepsilon^{l}+\varepsilon^{l} \varepsilon^{0}\right) H_{1} \partial_{l} } \\
& +\varepsilon^{l} \varepsilon^{l^{\prime}} H_{1} \partial_{l} \partial_{l^{\prime}}+\left(\varepsilon^{0} \varepsilon^{p} H_{1}+\varepsilon^{p} H_{1} \varepsilon^{0}+\varepsilon^{p} \varepsilon^{0} H_{1}\right) H_{1} \\
& +\varepsilon^{l} \varepsilon^{0} H_{1}^{2} \partial_{l}+\varepsilon^{p} \varepsilon^{l} H_{1} \partial_{l} H_{1}+\varepsilon^{p} H_{1} \varepsilon^{l} H_{1} \partial_{l} \\
& +\varepsilon^{p}\left(H_{1} \varepsilon^{p}+\varepsilon^{p} H_{1}\right) H_{1}^{2}+o\left(\varepsilon^{2}\right),
\end{aligned}
$$

where, as before, $W_{j}=W_{j}^{q}, \varepsilon^{a}=\varepsilon^{q a}$, etc. From the point of view of the order with respect to the interaction term $H_{\text {int }}$ of the hamiltonian, the expression (45) for $W^{2}$ can be rewritten as

$W_{2}=A_{2}\left(H_{\text {int }}\right)+A_{4}\left(H_{\text {int }}\right)+A_{6}\left(H_{\text {int }}\right)+o\left(\varepsilon^{2}\right)$,

where

$$
\begin{aligned}
A_{2}\left(H_{\mathrm{int}}\right)= & \left(I_{v}+\varepsilon^{0}\right) H_{1}+\varepsilon^{l}\left(H_{1} \partial_{l}+\varepsilon^{l^{\prime}} H_{1} \partial_{l} \partial_{l^{\prime}}\right), \\
A_{4}\left(H_{\mathrm{int}}\right)= & \left(\varepsilon^{0} \varepsilon^{l}+\varepsilon^{l} \varepsilon^{0}\right) H_{1} \partial_{l}+\varepsilon^{p}\left(\varepsilon^{l} H_{1} \partial_{l} H_{1}+\varepsilon^{l} H_{1} \partial_{l}\right), \\
A_{6}\left(H_{\mathrm{int}}\right)= & \left(\varepsilon^{0}\right)^{2} H_{1}+\left(\varepsilon^{0} \varepsilon^{p} H_{1}+\varepsilon^{p} H_{1} \varepsilon^{0}+\varepsilon^{p} \varepsilon^{0} H_{1}\right) H_{1} \\
& +\varepsilon^{l} \varepsilon^{0} H_{1}^{2} \partial_{l}+\varepsilon^{p}\left(H_{1} \varepsilon^{p}+\varepsilon^{p} H_{1}\right) H_{1}^{2} ;
\end{aligned}
$$

$A_{2}$ contains terms of the second order, $A_{4}$ - of the second and fourth orders, and $A_{6}-$ of the second, fourth and sixth orders with respect to $\left|H_{\text {int }}\right|^{\frac{1}{2}}$ or, equivalently, with respect to the operators of creation and annihilation of electrons.

Indeed, the expressions obtained for the energy $W=\sum W^{q}$ of the system of electrons, placed in a field characterized by the geometry corresponding to the pseudo-riemannian Hurwitz pair $\left(\mathbf{B}_{\mathrm{M}}, V\right)$ in question, provide us with a starting point for describing the system in terms of the second quantization [21]. To this end, in a usual way, we associate with the energy $W$ the hamiltonian $H$ determined with respect to the operators of creation and annihilation of electrons via expanding the wave function into a series of eigenfunctions constituting system of solutions of the generalised Breit equation (33), being at the same time a general Dirac-Maxwell system, in the one-electron approximation. The structure of the hamiltonian $H$, yielded by the energy $W$ approximated with the exactness of $o\left(\varepsilon^{2}\right)$ by $W_{2}=\sum W_{2}^{q}$ as given by (46), shows that $H$ contains terms of the second, fourth and higher orders with respect to the operators of annihilation and creation of electrons: for instance, the addends composing the term $A_{6}$ in (47) for $W_{2}$ generate contributions of the second, fourth and sixth orders in the expression for $H$.

The basic addends in the interaction matrix elements contain the terms known from the nonrelativistic theory, but the interpolation of one- and two-electron interactions indicate their more complicated character and the appearance of interactions of the relativistic and geometrical-deformational origin. The terms of the sixth and higher orders with respect to the annihilation and creation operators are of a particular interest; they naturally follow from the given theory as terms corresponding to the simultaneous interaction of more than two electrons and have a characteristic significance from the viewpoint of statistical estimation for interactions of the particle system. This fact indicates the dependence of the band structure on temperature, implied by electron interactions of a collision type and by the interchange of spin orientations. Of course, the established temperature dependence is quite different in its character from that usually taken into account, influenced by oscillations of the crystallographic lattice [6].

Following the usual procedure of the second quantization [21], we arrive at an expression for the hamiltonian $H$ associated with the energy $W$, which is of the 
form

$$
\begin{aligned}
H= & \sum_{\lambda, \lambda^{\prime}} t_{\lambda \lambda^{\prime}} a_{\lambda}^{+} a_{\lambda^{\prime}}+\frac{1}{2} \sum_{\lambda \varrho \varrho^{\prime} \lambda^{\prime}} I_{\lambda \varrho \varrho^{\prime} \lambda^{\prime}} a_{\lambda}^{+} a_{\varrho}^{+} a_{\varrho^{\prime}} a_{\lambda^{\prime}} \\
& +\frac{1}{4} \sum_{\lambda \varrho \times x^{\prime} \varrho^{\prime} \lambda^{\prime}} L_{\lambda \varrho \times x^{\prime} \varrho^{\prime} \lambda^{\prime}} a_{\lambda}^{+} a_{\varrho}^{+} a_{\varkappa}^{+} a_{\varkappa^{\prime}} a_{\varrho^{\prime}} a_{\lambda^{\prime}} \\
& +o\left(\left\|a_{\lambda}^{+} a_{\lambda^{\prime}}\right\|^{3}\right),
\end{aligned}
$$

where $a_{\lambda}^{+}$resp. $a_{\lambda}$ denote the creation resp. annihilation operators, while the matrix elements $t_{\lambda \lambda^{\prime}}, I_{\lambda \varrho Q^{\prime} \lambda^{\prime}}$ and $L_{\lambda \varrho \times x^{\prime} \ell^{\prime} \lambda^{\prime}}$ contain the one-, two- and threeparticle interactions, chosen according to (47). We have to notice that $A_{4}$ gives the tems of the second and fourth orders, while $A_{6}$ the terms of the second, fourth and sixth orders.

An analysis of (47) shows that the linear dependence of the energy of the hyperfine interactions on the values of electrons and nuclear spins holds with the exactness to $o(\varepsilon)$, thus proving with that exactness the theorem of Oguchi; the parameters $\varepsilon^{a}$ permit to estimate the influence of higher orders' terms, but it is interesting that in strong fields there may occur nonlinear interactions, forseen in a natural way by the theory.

The form (47) of the energy $W$ and the related form (48) of the hamiltonian $H$ indicate also the appearance of corrections to the electron correlation functions, describing the electron states density, which yields a change of the charge density distribution, the work function, and the other parameters characterizing the system of electrons. In particular, the autocorrelation of electrons, describing the behaviour of an electron at two different instants, expresses a delocalization of that electron, pointing out a finite life time of the definite quantum state of the electron. As an interesting example we may here mention the reversing of the spin orientation and the autocorrelation time which describe the blurring of that process. In the nonrelativistic theory, the spin autocorrelation corresponding to the two-particle autocorrelation of the type

$$
\begin{aligned}
\exp \left(i \frac{H}{\hbar} t\right)\left(a_{r \uparrow}^{+} a_{r \uparrow}-a_{r \downarrow}^{+} a_{r \downarrow}\right) \\
\quad \cdot \exp \left(-i \frac{H}{\hbar} t\right)\left(a_{r \downarrow}^{+} a_{r \uparrow}-a_{r \uparrow}^{+} a_{r \downarrow}\right)
\end{aligned}
$$

is determined by a diffusion-type equation. When including a relativistic approach connected with the hamiltonian (48), in particular the dependence on $A_{6}$ and following addends in (47), we will arrive at establishing the influence of the higher order correlation on the relaxation processes and hence on the spectrum lines widening, implemented by the autocorrelation time as well. That influence is of a more complicated character since it depends intrinsically on the temperature via the occurrence of many-particle interactions.

\section{Conclusions}

The relativistic approach to the many-electron problem as a generalisation of the Breit equation in the geometry corresponding to a Hurwitz pair $\left(\mathbf{B}_{\mathrm{M}}, V\right)$ with an arbitrary pseudo-riemannian metric $g$ leads to an effective hamiltonian $H$, given by (48), acting in the space of observations $\mathbb{M}$. The hamiltonian includes a number of interactions being a consequence of the supposed space-time in the spirit of the Kałuża-Klein theory of an arbitrary order $p=8 k+5$, where $k+1$ denotes the number of electrons in question. In the roughest approximation corresponding to the infinite velocity of interactions, $H$ reduces to the well-kown nonrelativistic form as the direct counterpart of the Schrödinger equation in terms of the second quantization. The interactions occurring, which correspond to the relativistic notion of space-time, can be identified with the respective terms appearing in the development of the Dirac equation or, more precisely, of the Dirac-Maxwell system.

Our proposal for the generalised Breit equation (33), including the pseudo-riemannian geometry and the Clifford structures in the form of Hurwitz pairs, leads to further types of interactions which are connected with the richness of the Clifford algebras, reflecting the intrinsic symmetries of the system. This confirms and mathematically justifies the fact that interactions within a particle system are not only a simple sum of interparticles, but also depend on the symmetry (more precisely: broken symmetry) properties of the space in which the particles occur (in our formalism: of the space created by the particles with all physical parameters like mass, charges, etc., intrinsically included in the pseudo-riemannian Hurwitz pair geometry $[2,3,10])$.

In the case of two particles $(k=1)$, in contrast to the usual Pauli theory [16], instead of two systems of spins we have $2^{4 k}=16$ systems of reduced spins $\left(\sigma_{l}^{q}\right)$, $q=1, \ldots, 16$. Two of them, corresponding e.g. to two electrons, are introduced by the equalities (43); the others corresponding to 14 types of interactions, may be defined analogously. This difference in approach 
has an important consequence: the $H_{\text {int }}$ contribution to the Dirac-like hamiltonian remains unchanged when passing to the Schrödinger-like expression (40) for the energy $W=\sum W^{q}$. Consequently, in spite of a considerable increase of the number of unknowns, from the computational point of view the new system of differential equations is not more complicated than (33), the initial one.

Next, in the light of our previous papers [22] we can see that the Dirac-like system of (33) always generates a generalised Kadomtsev-Petviashvili system of differential equations having, in general, soliton solutions. Namely, let us consider the operators $\tilde{D}^{q}$ in (33), determined by (36). We associate with them pseudo-differential operators

$$
\begin{array}{r}
W_{q}(t)=I_{v}+u_{q}^{1}(z, t)\left(\tilde{D}^{q}\right)^{-1}+u_{q}^{2}(z, t)\left(\tilde{D}^{q}\right)^{-2}+\ldots, \\
z \in \mathbb{M},
\end{array}
$$

where $t=\left(t_{1}, t_{2}, \ldots\right)$ is a system of infinitely many parameters. We set $L_{q}(t)=W_{q} \widetilde{D}^{q} W_{q}^{-1}$ (not being summed). Then we can see that $L_{q}(t)$ determines an isospectral deformation of $\widetilde{D}^{q}$ : any eigenfunction $\lambda_{q}$ of the equation $L_{q}(t) \Psi=\lambda_{q} \Psi$ remains fixed, independently of the choice of $t$. The corresponding Kadomtsev-Petviashvili system reads

$$
\frac{\partial}{\partial t_{n}} L_{q}=\left[\left(L_{q}^{n}\right)_{+}, \mathrm{L}_{q}\right], \quad n=1,2, \ldots,
$$

where

$$
\left(L_{q}^{n}\right)_{+}=\left(\frac{\partial}{\partial t_{n}} W_{q}\right) W_{q}^{-1}+L_{q}^{n},
$$

and $L^{n}$ is defined as $L^{n-1} L$.

Therefore, when inserting to the hamiltonian the variational expressions of the type (20) for the metric and of the type (28) for the spinor connections, we may utilize the method of isospectral deformations related to (49). In terms of Hurwitz pairs and the related supercomplex structures [12], this method is reflected by the theorem stating that, given a Hurwitz pair $\left(\mathbf{B}_{\mathrm{M}}, V\right.$ ), a deformation of any complex structure $J$ (in the usual sense) of the complexified vector space $V$ is isospectral if and only if the deformed Hurwitz pair has the same normalized related Clifford algebra in the sense of Theorem 1 in Ref. [17], determined via the eigenvalue $z$ of the endomorphism $J$, being a point of the fibre space $\mathbb{M}$ :

$J f=z \cdot f$ for $f \in V$, where $:: T_{z} \mathbb{M} \times V \rightarrow V$.
The theorem can still be generalised by replacing that point by any point of a suitably chosen Stiefel manifold of dimension $p-1$; cf. Refs. [23, 12].

The general considerations connected with the problem in question let us also formulate some conclusions concerning the concrete characteristics of the system. One of them is the self-consistency of the electromagnetic field and its connection with the shape of the metric. As forseen in our previous papers [2, 3, 11], the formulae (44) for the energy and (48) for the effective hamiltonian imply the occurrence of the electromagnetic field generated by the dipole interactions which come from the spin orientations characterized by the generators of Clifford algebras being a generalisation of the Dirac matrices. In the case of a system of electrons, bounded by a surface, the flux of the electromagnetic field, manifesting itself as a deformation of the space outside the object, determines in a natural way the demagnetizing fields existing in the region over the surface. The occurrence of those fields, experimentally measurable, enables us to determine the boundary conditions for solutions describing the electron density distribution inside the system. In the sense of the geometrical approach to the problem, the boundary conditions can be expressed via deformation of the metric in the region close to the surface.

The latter conclusion can be joined with the applicability of the theorem of Goldstone [24] referring to the mass spectrum of the particles, with a description of systems of the skyrmions [25]. That description is also intrinsically related to the machinery of Clifford algebras and analyses, and thus leading, in particular, to equations of the Kadomtsev-Petviashvili type with soliton solutions $[22,26]$. From the viewpoint of the imposed boundary conditions we can generalise the causes of occurrence of the boson particles; namely, they reduce either to the lack of deformation or to a sufficiently strong deformation manifesting itself outside the crystal by solitary waves. In this sense a deformation of the symmetry of interactions, i.e. the symmetry breaking, and a suitably preassumed deformation of geometry or, more generally, also of the related supercomplex structure, are mutually equivalent.

The above conclusions show that the present paper gives a further development of the research programme initiated in [5] and then continued in [3]. 
[1] J. Ławrynowicz and L. Wojtczak, Z. Naturforsch. 29a, 1407 (1974).

[2] J. Ławrynowicz and L. Wojtczak, Z. Naturforsch. 32 a, 1215 (1977).

[3] J. Ławrynowicz and L. Wojtczak, Rev. Roumaine Math. Pures Appl. 33, 89 (1988).

[4] B. Gaveau, J. Ławrynowicz, and L. Wojtczak, in: Mathematical Structures - Computational Mathematics Mathematical Modelling II. Papers dedicated to Professor L. Iliev's 70th anniversary, Publ. House of the Bulgarian Acad. of Sciences, Sofia 1984, p. 70.

[5] B. Gaveau and G. Laville, Bull. Soc. Sci, Letters Łódź 32, 1 (1982).

[6] B. Gaveau, J. Lawrynowicz, and L. Wojtczak, Phys. Stat. Sol. (b) 121, 47 (1984).

[7] J. Milnor, L'Enseignement Math. (2), 9, 198 (1963).

[8] T. Kałuża, Sitzungsberichte der Berliner Akademie der Wissenschaft, Math.-Phys. Klasse 1921, 966 (1921). O. Klein, Z. Physik 37, 895 (1926).

[9] H. Lee (ed.), An Introduction to Kaluza-Klein Theories, World Scientific, Singapore 1984.

[10] J. Ławrynowicz and J. Rembieliński, J. Phys. A: Math. Gen. 20, 5831 (1987).

[11] J. Ławrynowicz, in: Séminaire P. Lelong, H. Skoda (Analyse) 1980/81. Lecture Notes in Math. 919, Springer, Berlin-Heidelberg-New York 1982, p. 152.

[12] J. Ławrynowicz and J. Rembieliński, in: Seminari di Geometria 1984 (S. Coen, ed.), Univ. di Bologna, Bologna 1985 , p. 131.

[13] J. Ławrynowicz, S. Koshi, and O. Suzuki, to be published.

[14] J. Ławrynowicz, in: Equadiff 78, Firenze 24/30 maggio 1978 (da R. Conti, G. Sestini, and G. Villari, eds.), CNR, Firenze 1978, p. 351.

[15] B. Gaveau, J. Ławrynowicz, and L. Wojtczak, in: Conf. Analytic Functions Abstracts, Blażejewko 1982, University of Łódź, Łódź 1982, p. 12,
[16] H. A. Bethe and E. E. Salpeter, Quantum Mechanics of One- and Two-electron Atoms, Springer-Verlag, Berlin 1957.

[17] J. Ławrynowicz, M. Porter, E. Ramirez de Arellano, and J. Rembieliński, Bol. Soc. Mat. Mexicana 34 (1989), in press.

[18] B. Gaveau, J. Kalina, J. Ławrynowicz, and L. Wojtczak, Ann. Polon. Math. 46, 105 (1986). - T. Eguchi, P. B. Gilkey, and A. J. Hanson, Phys. Rep. 66, 213 (1980).

[19] Z. Oguchi, in: Proc. Summer School of Crit. Phen., Cetniewo (Poland) 1973, p. 95. - E. Bradford and W. Marshall, Proc. Phys. Soc. London 87, 731 (1966).

[20] W. Gabriel, Phys. Rev. 181, 506 (1969). - A. Kobeissi, R. Suler, A. Gottlieb, and C. Hohenemser, Phys. Rev. 311, 2455 (1975). - A. Urbaniak-Kucharczyk, Phys. Stat. Sol. (b) 109, K 59 (1982); 139, K 141 (1987).

[21] A. L. Fetter and J. D. Walecka, Quantum Theory of Many-Particle Systems, McGraw-Hill, New York 1971.

[22] J. Kalina, J. Lawrynowicz, and O. Suzuki, Suppl. Rend. Circ. Mat. Palermo (2) 9, 117 (1985); Rend. Sem. Fac. Sci. Univ. Cagliari 57, 131 (1987). - O. Suzuki, J. Ławrynowicz, J. Kalina, and S. Kanemaki, Proc. Inst. Nat. Sci. College Hum. Sci. Nihon Univ. 21, 11 (1986); 24 (1989).

[23] J. Ławrynowicz and J. Rembieliński, in: Seminar on Deformations, Proceedings, Łódź-Warsaw 1982/84. Lecture Notes in Math. 1165, Springer, Berlin 1985, p. 184.

[24] A. P. Balachandran, F. Lizzi, V. G. J. Rodgers, and A. Stern, Nucl. Phys. B 256, 525 (1985). - A. P. Balachandran, in: High Energy Physics 1985 (M. J. Bowick and F. Gürsey, eds.), World Scientific, Singapore 1986, p.1.

[25] H. R. Skyrme, Proc. Roy. Soc. London Ser. A 260, 127 (1961); 262, 237 (1961); Nucl. Phys. 31, 556 (1962).

[26] A. Chodos, E. Hadjimichael, and C. Tze (eds.), Solitons in Nuclear and Elementary Particle Physics, World Scientific, Singapore 1984, p. 1. 